\title{
Investigation On Probing Schemes In Probe-Based Multicast Admission Control
}

\author{
Chunhui Le Jianhua He Guanxiang Zhang and Wenqing Cheng \\ Huazhong University of Science and Technology, \\ Wuhan City, 430074, P. R. China
}

\begin{abstract}
Multicast is an efficient approach to save network bandwidth for multimedia streaming services. To provide Quality of Services (QoS) for the multimedia services while maintain the advantage of multicast in bandwidth efficiency, admission control for multicast sessions are expected. Probe-based multicast admission control (PBMAC) schemes are of a sort of scalable and simple admission control for multicast. Probing scheme is the essence of PBMAC. In this paper, after a detailed survey on three existing probing schemes, we evaluate these schemes using simulation and analysis approaches in two aspects: admission correctness and group scalability. Admission correctness of the schemes is compared by simulation investigation. Analytical models for group scalability are derived, and validated by simulation results. The evaluation results illustrate the advantages and weaknesses of each scheme, which are helpful for people to choose proper probing scheme for network.
\end{abstract}

Keywords: Q uality of Service, Multicast, Admission Control, Multimedia Communication

\section{INTRODUCTION}

Multicast technique has been widely studied during the past decade. It is viewed as an efficient approach to save network bandwidth for multimedia streaming services. However, multimedia streaming services normally have stringent QoS requirements. To provide QoS for the services while maintain the advantage of multicast on bandwidth efficiency, admission control for multicast sessions is expected to control the total traffic load of the network. However, the problem of multicast admission control was much less investigated. The existing multicast admission control schemes can be classified into two broad categories. The first one is developed from classic unicast measurement-based admission control, combined with RSVP, to reserve bandwidth hop by hop from the receiver to the graft point of the multicast tree. ${ }^{1}$ The idea is that each router make its admission decision independently based on its measured available resource. Once the flow is admitted, the resource will be reserved for it. A connection will be established if all nodes admit. As each router is required to make local admission decision, this scheme is not scalable and not suitable for large scale deployment of multicast services in Internet. The second type of multicast admission control scheme is probe-based, which is called PBMAC in our paper. Karlsson and Elena proposed schemes of this category respectively ${ }^{2}{ }^{3}$ PBMAC borrows the idea from probe-based unicast admission control, which received many research efforts recently ${ }^{4}{ }^{5}$ Unlike unicast admission control, admission requests are initiated by the receivers in multicast. A host probes network to find whether the available bandwidth is sufficient for its requirement before joining a new session and receiving data. The probe traffic has a lower priority $P r_{p}$ than that of data traffic (noted with $P r_{d}$ ), thus the probing process will not affect QoS perceived by existing multicast sessions. Without keeping per-flow states in the routers, the probe-based scheme achieves high scalability and is easy to deploy.

The essence of PBMAC is the probing scheme. We category the probing scheme in three types: additional peak rate probing (APRP), data probing (DP) and complementary peak rate probing (CPRP). In APRP, ${ }^{2}$ probe traffic is sent at the peak data rate, additional to the data traffic. In $\mathrm{DP},{ }^{3}$ data traffic is remarked to a lower forwarding priority and used to probe the newly grafted multicast branches, which is called in this paper. $\mathrm{CPRP}^{6}$ is A new proposed scheme. In this scheme, complementary traffic with low priority, together with low

Further author information: (Send correspondence to Chunhui Le)

Chunhui Le: E-mail: lechunhui@hust.edu.cn, Telephone: 862787453207 
remarked data traffic, is used to probe the newly grafted multicast branches. In the next section, three probing schemes are surveyed in more details.

There is a tradeoff between admission correctness and bandwidth utilization in admission control problem. In this paper, analytical models are proposed to gain an insight into these schemes. Simulations are also carried out in different scenarios for the investigation.

The rest of the paper is organized as follows. Three probing schemes are surveyed in Section 2. In Section 3, the admission correctness of these schemes are investigated by simulation. In Section 4, we evaluate the group scalability of probing schemes via analysis and simulation using metric - Normalized Requested Equivalent Link Capacity. We draw conclusion in the last section.

\section{SURVEY ON PROBE-BASED ADMISSION SCHEMES}

\subsection{Additional Peak Rate Probing}

Karlsson proposed a probe-based admission control scheme for multicast. ${ }^{2}$ In this scheme, a multicast group named probe group is created, associated with each multicast group delivering the actual data (called data group). Traffic for the probe group has lower priority. Users intending to join the data group are required to join the probe group first, and take a probing process. In the probe group, the multicast source sends probes at the peak data rate $R_{p}$ with priority $\operatorname{Pr}_{p}$ which is lower than data priority $\operatorname{Pr}_{d}$ to prevent the QoS violation of admitted sessions. The probing process for each receiver lasts for a certain time. During the process, the receiver receives probe packets and measures the loss ratio, which can be achieved by using sequence number in probe packets. After the probing process, the receiver leaves the probe group and admission decision is made based on measured packet loss ratio. If the loss ratio does not exceed the given threshold, the receiver is allowed to join the data group; otherwise, the receiver is rejected.

In this scheme, the probe traffic is sent at the peak data rate in addition to the data traffic. Hence, we name it additional peak rate probing (APRP) in this paper. The scheme is scalable, as well as easy to implement. And peak rate probing guarantees the confident level of admission to a high value. However, the Subsequent Request Problem occurs in this scheme, which may restrict the group size of multicast sessions in heavy-loaded network. ${ }^{6}$

If the multicast data traffic is being delivered over link $L$ due to the successful admission of request $A$ when probing process for request $B$ starts, we call request $B$ a subsequent request over link $L$, and $L$ the shared link of request $A$ and $B$. It's clear that the admission of a subsequent request over the link will not cost any extra resources on $L$. However, in APRP, when the traffic on the bottleneck link is close to its admissible level, the blocking probability of the subsequent requests may be extremely high. We call this problem subsequent requests problem.

The cause of subsequent request problem is the co-existence of the probe traffic and the data traffic on the bottleneck link. In APRP, when a subsequent joining request arrives, probe traffic is sent to the receiver through the bottleneck link where data traffic exists, which requires much more extra bandwidth. If the available bandwidth is not sufficient for the probe traffic, probes will experience a high loss, which results in high request blocking probability.

\subsection{CAMP and Data Probing}

An end-to-end Call Admission Multicast Protocol (CAMP) was proposed by Elena, et al. ${ }^{3}$ Like the preceding scheme, CAMP operates on-demand; it is scalable and supports the dynamic changes of the multicast group membership. A proxy mechanism is used in CAMP. The proxy lifetime lasts throughout the set-up phase. The proxy uses the information in the local routing table to maintain a data structure containing the list of downstream multicast interfaces (oifs), the list of destinations for each oif and the oif state. Initially, all the oifs in the table are in the probing state. During its activity, all the incoming packets for the session, which must be forwarded to the probing oifs, are remarked as probe packets by the proxy. The proxy labels the forwarded packets with its own address. The receivers send their reports to their proxies.

In CAMP, the data packets are remarked to a low priority at the graft point by the proxy and forwarded to the requesting receiver to probe the unknown links. We call this probing scheme data probing (DP). Since 
data traffic is remarked and used for link probing, the co-existence of data traffic and probe traffic is eliminated, and the Subsequent Request Problem is avoided. In the literature, it is illustrated that CAMP achieves good performance in CBR traffic scenario. However, we found that, with VBR traffic, the variation of throughput may cause the inaccuracy in link state estimation, and results in over admission of new multicast sessions. We'll analyze the problem in later sections.

\subsection{EPBMAC and Complementary Peak Rate Probing}

Considering subsequent request problem in APRP, complementary peak rate probing is proposed to enhance PBMAC. ${ }^{6}$ EPBMAC inherits the basic idea of Karlsson's admission scheme. A multicast source creates a multicast data group and a probe group, and traffic of probe group is marked to a lower priority $P r_{p}$ than that of data group traffic $\left(P r_{d}\right)$. Traffic at the peak data rate with low priority $P r_{p}$ is used in EPBMAC to probe the new multicast branch. However, in EPBMAC, complementary peak rate probing scheme (CPRP) is used on the shared links, and remarking operation is executed on the node at the graft point of the multicast tree for the new receiver.

$\mathrm{CPRP}$ is a compromise of APRP and DP, which takes advantages from both data traffic remarking mechanism in data probing and peak rate probing in addition to peak rate probing. In CPRP, the traffic used to probe the newly grafted multicast branch is composed of two parts: basic probe traffic $F_{p e}$ and additional probe traffic $F_{p d} . F_{p e}$ is generated by the multicast source and sent to the probe group. It is complementary to the data traffic, i.e., the source sends the probe traffic at rate $R_{p e}(t)$ at time $t$ :

$$
R_{p e}(t)=R_{p k}-R_{d}(t)
$$

where $R_{p k}$ is the peak rate of the data group and $R_{d}(t)$ is the data rate at time $t . F_{p d}$ is actually the traffic of data group, but it is remarked with the priority $P r_{p}$ at the graft point of the new branch. Hence, $F_{p e}$ is also complementary to $F_{p d}$.

By this means, the total traffic of probe and data keeps at the peak rate of the data flow. On newly grafted links, the total probe traffic (sum of $F_{p e}$ and $F_{p d}$ ) keeps at the constant rate $R_{p}$, while on the shared links, the total session traffic rate remains no more than $R_{p}$.

\section{ADMISSION CORRECTNESS}

Correctness is the basic requirement for admission. When error admission occurs, not only the wrongly admitted connection cannot meet its QoS requirement, but QoS of all the crossing traffic will be violated. For this sake, most admission control schemes make conservative decision in critical range of traffic load.

In PBMAC, as admission decision is made only based on the probe result, the probing approach determines the admission correctness. As introduced in the previous section, peak rate probing schemes (including APRP and CPRP) tries to create the worst case that the receiver may experience in future data receiving by probing the branch to graft into the multicast tree with traffic at peak data rate. Whereas, in data probing scheme, real data is employed to do probing. In fact, PBMAC and PBAC for unicast are almost the same on admission correctness issue. The only difference is that in multicast admission control, only newly grafted branch is probed before admission decision, while in unicast admission control, the whole path from source to the destination is probed. This makes no essential distinction. However, no comparison of peak rate probing and data probing for unicast has been found in literatures. Therefore, we'll not differentiate between multicast and unicast in the rest of the section.

For VBR traffic, especially video stream, the data rate may vary large. Since the probing period should not last too long, the data traffic used for probing may not cover the case that highest bandwidth is required. In core network, there are a large number of flows. The variance of background traffic may be so greatly that we can ignore the change of a single micro flow's throughput. However, in access network, which owns less bandwidth and fewer connections, the throughput variance of the requesting session is not ignorable. In this case, data probing is more probable to make an over-optimistical admission decision than peak rate probing when the link in access network is congested. 
In this section, we study the admission correctness by simulations. Since the traffic variance of VBR source has larger impacts on the probing result over access network, we focus on this issue and design scenarios to simulate this case.

To evaluate the admission correctness, we employ error admission probability. For specific flow req in particular scenario, error admission probability err is defined as $\operatorname{Prob}\{r e q$ is not admissive. $\mid r e q$ is admitted. $\}$. In our simulation investigation, empirical error admission probability $\widehat{e r r}$ is employed, in stead. The empirical error admission probability is computed as follows.

$$
\widehat{e r r}=\frac{\text { Num }}{\text { error }},
$$

where Num $_{\text {error }}$ denotes the number of admitted requests in error, and Numadmit is the total admitted request number.

Figure 1 illustrates the topology employed in the simulation. Sources $S_{1}-S_{n}$ send traffics to downstream network via bottleneck link $L$. In simulations for correctness investigation, the source number $n$ is 8 . Among these sources, $S_{1}-S_{7}$ are configured to generate data packets with high priority as the background traffic. $S_{8}$ is the source of the multicast session that a new receiver intends to join. It sends different types of traffic in different simulation steps. The data packet size is $1.5 \mathrm{~KB}$. At each source, the inter packet time follows Pareto distribution with the location parameter 0.01 and shape parameter 1.5, i.e., the data traffic of source possesses peak rate $1.2 \mathrm{Mbps}$ and mean rate $400 \mathrm{Kbps}$. The total bandwidth of the bottleneck link $L$ is $4.8 \mathrm{Mbps}$. For each join request, probing period lasts for 5 seconds. The target loss ratio is 0.01 . To simplify the development, probe packet size of each scheme is same to the data packet size in our simulations on admission correctness, though actually, small packets are used for probe traffic in APRP and basic probing traffic in CPRP.

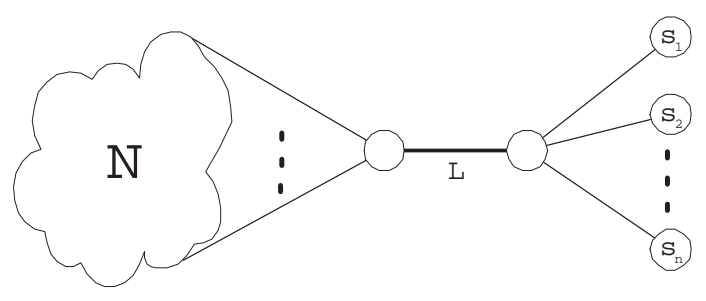

Figure 1. Network Topology of Simulations

In the first step, $S_{8}$ sends probe traffic with lower priority and the rate conforming to different probing schemes. The receiver estimates the loss ratio every second based on the received and lost packets in the last 5 seconds. The simulation last for 10 minutes. Figure 2 depicts the curve of cumulative distribution function (CDF) of each probing schemes which is obtained in the simulations. The figure shows that, in the same scenario, admission probability of data probing is about $5 \%$, while that of peak rate probing is almost 0 .

In the second step, $S_{8}$ sends data traffic as other sources. The sources generate data traffic exactly same to that in the first step. Loss ratio of the total traffic, as well as that of each flows, is measured. Figure 3 shows the result. Hence, the admission correctness in the first step is examined. In Figure 3, the overall loss ratio excesses the threshold 0.01 during some periods, and the QoS (loss ratio) violation of some particular flows is even worse. The posteriori results shows that the new request(connection to $S_{8}$ ) should not be admitted.

Combining results of the two steps of simulation, we conclude that peak rate probing achieves higher correctness than data probing.

\section{GROUP SCALABILITY}

\subsection{Analytical Modelling}

To simplify the analysis of the problem, we suppose there is only multicast traffic in the network. Let $\mu$ denote the bandwidth of the bottleneck link we consider, and $n$ denote the number of multicast sessions on the considered link.

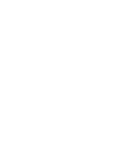




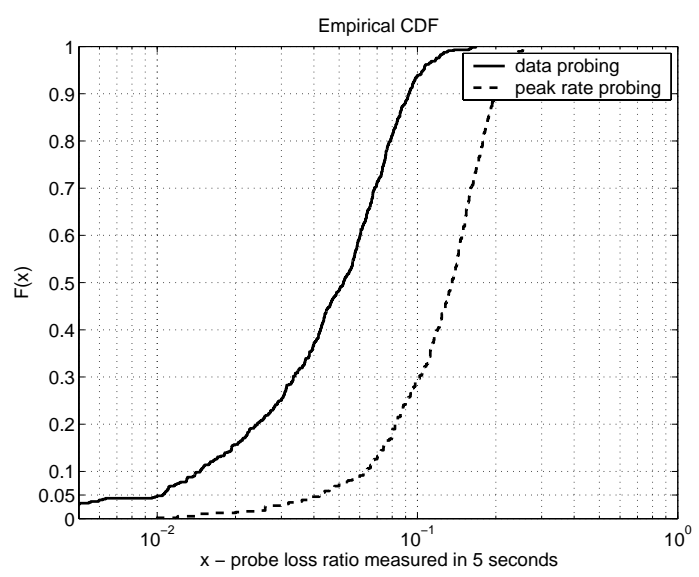

Figure 2. CDF of Probe Loss Ratio

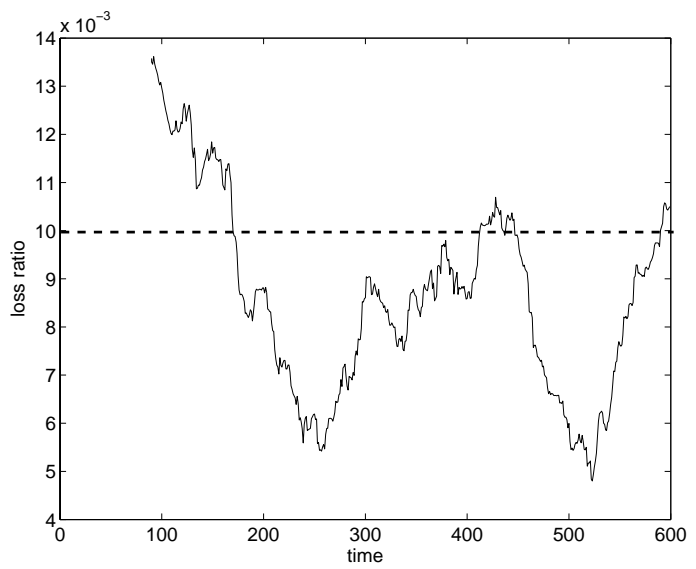

(a) Overall Loss Ratio on Link $L$

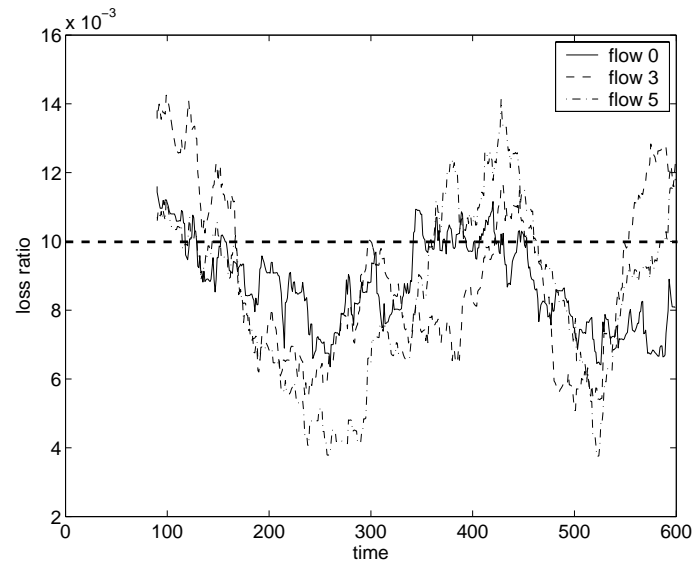

(b) Flow Loss Ratio

Figure 3. Empirical Loss Ratio - The time window of loss ratio measurement is 90 seconds, the measurement is taken every second.

Group scalability is a particular performance metric for PBMAC. As stated in the previous section, the subsequent request problem occurs in some probing schemes, which constrains the group scalability. In this subsection, we'll provide analytical models of the problem for all three probing schemes.

\subsection{Normalized Requested Equivalent Link Capacity}

Before analysis, we define a new metric - normalized requested equivalent link capacity (NRELC in short) to evaluate the group scalability.

$$
C=\sum_{i=1}^{n} R_{p k}^{i} \cdot r_{r e q}^{i} / \mu
$$

Where $R_{p k}^{i}$ and $r_{r e q}^{i}$ are the peak data rate and the request arrival rate of the multicast session $i$ respectively. Supposing the holding time of group numbers keeps in a stable distribution with a mean $\bar{t}_{\text {hold }}^{i}$, the maximize group size $S i z e^{i}=\bar{t}_{\text {hold }}^{i} \cdot r_{r e q}^{i}$. Hence

$$
C=\sum_{i=1}^{n} R_{p k}^{i} \cdot S i z e^{i} /\left(\mu \bar{t}_{h o l d}^{i}\right)
$$


From (4), we know that, if $\mu \bar{t}_{\text {hold }}^{i}$ is fixed, NRELC is a weighted sum of group sizes over particular link. Hence, it well reflects the overall group scalability of the link. In the sequent text, $C^{A}, C^{D}$ and $C^{C}$ denote NRELC with APRP, DP and CPRP respectively.

For ease of traffic load evaluation, we also define normalized traffic load as follows.

$$
\nu=\sum_{i=1}^{n} R_{p k}^{i} / \mu .
$$

We assume in the following analysis that the data traffic of each session complies with the same two-state fluid model. Let $R_{p k}$ and $R_{m}$ denote the peak rate and the mean rate of each data session respectively. And let $\rho=R_{m} / R_{p k}$. It is also assumed that the probing period of a new joining request for each session lasts for the same time $\tau$, and the arrival of joining request for each session from downstream receivers of the bottleneck link is a Poisson process with the same mean rate $\lambda$.

In this scenario, Equation (5) is converted to

$$
\nu=n R_{p k} / \mu
$$

and Equation (3) is transformed to

$$
\begin{aligned}
C & =n R_{p k} \cdot \lambda / \mu \\
& =\lambda \cdot \nu
\end{aligned}
$$

Obviously,

$$
\left\{\begin{array}{l}
\nu \geq 0 \\
\nu<\frac{\mu}{R_{m}} \cdot \frac{R_{p k}}{\mu}=1 / \rho .
\end{array}\right.
$$

In the sequent subsections, we'll use NRELC as a metric to evaluate group scalability of each probing scheme.

\subsubsection{DP}

When $n$ is sufficiently large, Gaussian distribution approximates well the total traffic distribution. The total data traffic $T_{d}$ follows the normal distribution $N\left(n \rho R_{p k}, n R_{p k}^{2} \rho(1-\rho)\right)$.

For DP, since only remarked data traffic is used for probing and no special probe traffic is introduced in the network, total traffic $T^{D}=T_{d}$ follows the normal distribution $N\left(n \rho R_{p k}, n R_{p k}^{2} \rho(1-\rho)\right)$.

Suppose to guarantee the the target loss ratio, $E\left(T^{D}\right)+c \cdot \sqrt{\operatorname{Variance}\left(T^{D}\right)}$ is the required bandwidth for traffic $T^{D}$ (where $c$ is a constant determined by the target loss ratio), according to the admission criterion of PBMAC, subsequent requests will be admitted if

$$
\begin{aligned}
& E\left(T^{D}\right)+c \sqrt{\operatorname{Variance}\left(T^{D}\right)} \\
= & n \rho R_{p k}+c R_{p k} \sqrt{n \rho(1-\rho)} \\
\leq & \mu .
\end{aligned}
$$

i.e.,

$$
\rho+c \sqrt{\rho(1-\rho) / n} \leq \mu .
$$

The admission criterion 9 is not related with NRELC $C^{D}$. Hence, so long as 9 is satisfied, $\max C^{D}=\infty$. Furthermore, since $\rho$ and $c$ are independent to $n$, and much less than $n$, when $n$ is sufficiently large, $c \sqrt{\frac{\rho(1-\rho)}{n}}$ is close to 0 , and inequation (9) is approximated with the validity condition of (4), which always holds for any valid $\nu$. 


\subsubsection{Analytical Model for APRP}

In Karlsson's PBMAC scheme, as a session starts to deliver probe packets over the bottleneck link only when a downstream node of the link requests for connection, the probe traffic on this bottleneck link complies with the two-state bursty model with the peak rate $R_{p k}$. Define $\eta^{A}$ as the probability of a given probe flow being active on the link.

$$
\eta^{A}=1-e^{-\lambda \tau}
$$

The mean probe rate of a single multicast session is $\eta^{A} R_{p k}$. Similarly, the distribution of total probe traffic with APRP (denoted by $T_{p}^{A}$ ) is approximated with Gaussian distribution when $n$ is sufficiently large, i.e., $T_{p}^{A} \sim N\left(n \eta^{A} R_{p k}, n R_{p k} \eta^{A}\left(1-\eta^{A}\right)\right)$.

Let $T^{A}=T_{d}+T_{p}^{A}$ denote the total traffic with APRP. Since $T_{d}$ and $T_{p}^{A}$ are independent Gaussian distributed random variables, $T^{A}$ also complies with the Gaussian distribution. The total traffic $T^{A}$ follows

$$
T^{A} \sim N\left(n R_{p k}\left(\rho+\eta^{A}\right), n R_{p k}^{2}\left(\rho(1-\rho)+\eta^{A}\left(1-\eta^{A}\right)\right)\right)
$$

Suppose to guarantee the the target loss ratio, $E\left(T^{A}\right)+c \cdot \sqrt{\operatorname{Variance}\left(T^{A}\right)}$ is the required bandwidth for traffic $T^{A}$ (where $c$ is a constant determined by the target loss ratio), according to the admission criterion of PBMAC, subsequent requests will be admitted if

$$
\begin{aligned}
& E\left(T^{A}\right)+c \sqrt{\operatorname{Variance}\left(T^{A}\right)} \\
= & n \rho R_{p k}+n \eta^{A} R_{p k}+c R_{p k} \sqrt{n \rho(1-\rho)+n \eta^{A}\left(1-\eta^{A}\right)} \\
\leq & \mu .
\end{aligned}
$$

From (6)(12), we have

$$
\rho+\eta^{A}+c \sqrt{\frac{\rho(1-\rho)+\eta^{A}\left(1-\eta^{A}\right)}{n}} \leq \frac{1}{\nu}
$$

Since $\eta^{A}, \rho$ and $c$ are independent to $n$, and much less than $n$, when $n$ is sufficiently large, $c \sqrt{\frac{\rho(1-\rho)+\eta^{A}\left(1-\eta^{A}\right)}{n}}$ is close to 0 , and inequation (13) could be approximated with

$$
\rho+\eta^{A} \leq \frac{1}{\nu}
$$

Combining with (10) and (7), we have

$$
C^{A} \leq \begin{cases}\infty, & \text { if } 0 \leq \nu \leq \frac{1}{1+\rho} \\ -\frac{\nu}{\tau} \ln \left(1-\left(\frac{1}{\nu}-\rho\right)\right), & \text { if } \frac{1}{1+\rho}<\nu<\frac{1}{\rho}\end{cases}
$$

Inequation (15) shows that, for fixed heavy data traffic, the admissive NRELC $C^{A}$ may be constrained. When $C$ exceeds the limit, the subsequent joining requests will be blocked. As we know that the admission of a subsequent request doesn't consume any extra resource on the bottleneck link, this constraint is not necessary. Even worse, it limits the advantage of multicast. 


\subsubsection{Analytical Model for CPRP}

The analysis of group scalability for CPRP is similar to that for APRP. We define following events for a session on the bottleneck link :

$E A$. Sending data at rate $R_{p k}$,

if data source is active;

$E B$. Sending probe at rate $R_{p k}$,

if data source is inactive and at least one joining request arrives in last $\tau$ interval;

$E C$. Silence,

otherwise.

According to the supplemental probe mechanism and the two-state fluid assumption, these events are incompatible. As defined previously, $\operatorname{Prob}\{E A\}=\rho$. Define $\alpha=\operatorname{Prob}\{E B\}$. And we also define events $E B_{1}=$ \{The data source is inactive. $\}$ and $E B_{2}=\{$ At least one joining request arrives in last $\tau$ interval. $\}$. Since $E B_{1}$ and $E B_{2}$ are independent,

$$
\begin{aligned}
\alpha & =\operatorname{Prob}\{E B\} \\
& =\operatorname{Prob}\left\{E B_{1}\right\} \operatorname{Prob}\left\{E B_{2}\right\} \\
& =(1-\rho)\left(1-e^{-\lambda \tau}\right) .
\end{aligned}
$$

Let $T^{C}$ denote the total traffic of the multicast sessions,

$$
T^{C} \sim N\left(n(\rho+\alpha) R_{p k}, n R_{p k}^{2}(\alpha+\rho)(1-(\alpha+\rho))\right)
$$

the admission criteria is

$$
\begin{aligned}
& E\left(T^{C}\right)+c \sqrt{\operatorname{Variance}\left(T^{C}\right)} \\
= & n(\rho+\alpha) R_{p k}+c R_{p k} \sqrt{n(\alpha+\rho)(1-(\alpha+\rho))} \\
< & \mu .
\end{aligned}
$$

When $n$ is sufficiently large, from (6), (7), (16), (17) we have the admissible normalized requested equivalent link capacity for EPBMAC

$$
C^{C} \leq \begin{cases}\infty, & \text { if } 0 \leq \nu \leq 1 \\ -\frac{\nu}{\tau} \ln \left(1-\frac{1 / \nu-\rho}{1-\rho}\right), & \text { if } 1<\nu<\frac{1}{\rho}\end{cases}
$$

Analysis above shows that data probing scheme avoids the subsequent request problem, so that the limitation in group size is eliminated and NRELC could up to infinity. In both peak rate probing schemes, NRELC is constrained in heavy traffic load. However, CPRP greatly relaxes the constraint, and achieves better group scalability.

\subsection{Simulation Investigation}

We do Simulations with OPNET to validate the analytical models above. The network model for the simulations is depicted in Figure 1. The bandwidth of link $L$ is $20 \mathrm{Mbps}$ and the other links do not create any bottleneck. The duration of probing process lasts for 5 seconds and the probe packet (if it is employed) size is 64 bytes. The request arrival rate for each session follows Poisson process. ${ }^{7}$ The target loss ratio threshold for admission is $1 \%$.

On/off bursty sources are used to simulate the data traffic from a multicast source. The on/off traffic parameters are listed in Table 1. The distribution of on/off period conforms Pareto distribution with the shape parameter 1.5. The probing process of each multicast session for a joining request lasts for 5 seconds. The 


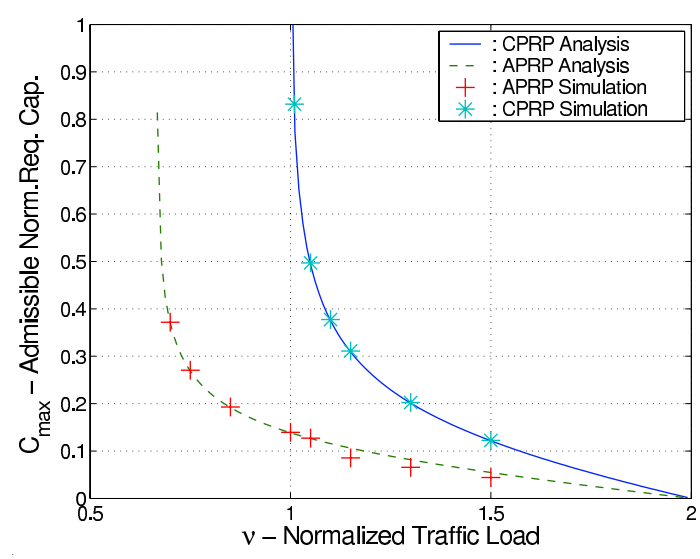

(a) Traffic Parameters Par1

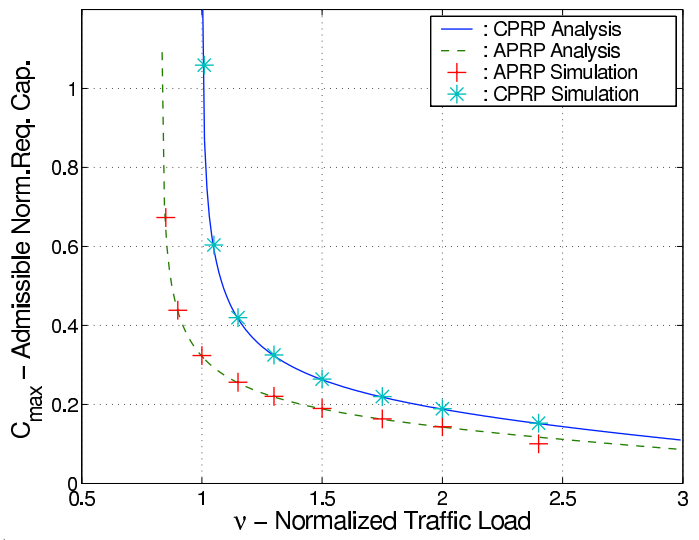

(b) Traffic Parameters Par2

Figure 4. $\nu-C_{\max }$ Curves of APRP and CPRP Based Simulation Results

request arrival rate for a session follows Poisson process. We change the mean rate of joining request arrival process $(\lambda)$ to find its maximum admissible value. The probe traffic depends on the joining request arrival rate and data traffic parameters. Normalized peak rate of each flow $p$, which is defined as $p=R_{p k} / \mu$, is set to 0.01 for the simulations and analytical curves. We alter the number of multicast sessions to change the bottleneck traffic. The maximum normalized equivalent requested link capacity $C_{\max }=\max \{C\}$ is computed according to (7) based on $\max \{\lambda\}$ measured in the simulation.

Table 1. Traffic Parameters in Simulations

\begin{tabular}{|c|c|c|}
\hline Traffic Type & Mean On Interval & Mean Off Interval \\
\hline Par1 & $20 \mathrm{~ms}$ & $80 \mathrm{~ms}$ \\
Par2 & $200 \mathrm{~ms}$ & $200 \mathrm{~ms}$ \\
\hline
\end{tabular}

Figure 4 presents typical simulation results of maximum admissible normalized equivalent requested link capacity $C_{\max }$ over normalized multicast traffic load $\nu$ of APRP and CPRP. The traffic parameters are listed in Table 1. The analytical curves for both APRP and CPRP are also plotted. It can be observed that the analytical results match well with the simulation results for both APRP and CPRP. It can also be observed that the performance of CPRP is evidently better than that of APRP in different types of traffic, and the simulation results fit well to the analysis.

\section{CONCLUSIONS}

PBMAC is a sort of scalable and simple admission control for multicast services, and probing scheme is the essence of PBMAC. In this paper, after a detailed survey on three existing probing schemes, namely APRP, DP and CPRP, we study these schemes by simulation and analysis in two aspects: admission correctness and group scalability.

Admission correctness is the basic requirement for an admission control scheme. The simulation investigation on admission correctness shows that the in critical traffic load range, peak rate probing schemes (APRP and DP) are more conservative, and make much fewer admission errors than DP.

Group scalability is a particular performance issue for PBMAC. We employ a new metric - NRELC to evaluate this feature. Analytical models and simulation results shows that subsequent request problem debases group scalability of APRP, CPRP can much release the problem by using complementary probing, and DP has the best performance in group scalability which avoids the constraint on NRELC. 


\section{REFERENCES}

1. R. Braden, L. Z. Ed., S. Berson, S. Herzog, and S. Jamin, "Resource reservation protocol (rsvp) - version 1 functional specification." IETF RFC 2205, Sept. 1997.

2. I. Mas, V. Fodor, and G. Karlsson, "Probe-based admission control for multicast," in Tenth IEEE International Workshop on Quality of Service 2002, pp. 15-17, May 2002.

3. P. Elena and R. G. Paolo, "Distributed bandwidth broker for qos multicast traffic," in Proc. of the 22nd International Conference on Distributed Computing Systems, pp. 319-326, July 2002.

4. L. Breslau, E. Knightly, S. Shenker, I. Stoica, and H. Zhang, "Endpoint admission control: Architectural issues and performance," in Proc. of ACM SIGCOMM 2000, (Stockholm, Sweden), Aug. 2000.

5. I. MsIvars and G. Karlsson, "Pbac: Probe-based admission control," in Proc. of QofIS 2001, 3, pp. 97-109, (Coimbra, Portugal), Sept. 2001.

6. C. Le, J. He, Z. Yang, and W. Liu, "An enhanced scalable probe-based multicast admission control scheme," Journal of Electronics (China), (Accepted).

7. K. Almeroth and M. Ammar, "Multicast group behavior in the internet's multicast backbone (mbone)," IEEE Communications Magazine 35, pp. 124 - 129, June 1997. 